\title{
Charmonium in electromagnetic and vortical fields
}

\author{
Jiaxing Zhao ${ }^{1, *}$, Shile Chen ${ }^{1}$, and Pengfei Zhuang ${ }^{1}$ \\ ${ }^{1}$ Physics Department, Tsinghua University, Beijing 100084, China
}

\begin{abstract}
Due to their large mass and early production, heavy quarks (quarkonia) can be sensitive probes to investigate the fast-decaying electromagnetic and vortical fields produced in heavy-ion collisions. The non-relativistic Schrödinger-like equation for heavy quarks under strong electromagnetic fields in the rotating frame is deduced and used to construct the two-body equation for the charmonium system. The effective potential between charm and anti-charm quarks becomes anisotropic in electromagnetic and vortical fields, especially along the direction of the Lorentz force. The vorticity will affect this asymmetry and catalyze the transition from a strong interaction dominant bound state to an electromagnetic and vortical interaction controlled anisotropic bound state.
\end{abstract}

Many theoretical studies show that strong electromagnetic fields are generated in noncentral relativistic heavy-ion collisions [1-5]. The maximum of the magnetic field can reach $5 m_{\pi}^{2}$ in semi-central $\mathrm{Au}+\mathrm{Au}$ collisions at top RHIC energy and almost $70 m_{\pi}^{2}$ in semi-central $\mathrm{Pb}+\mathrm{Pb}$ collisions at LHC energies $[4,5]$, where $m_{\pi}$ is the pion mass. In addition, there is a finite total angular momentum $J \propto b \sqrt{s_{N N}}$ ( $b$ is the impact parameter) carried by the system of two colliding nuclei in non-central heavy-ion collisions. Although most of this total angular momentum is carried away by the spectators, there is still a sizable fraction that remains in the created quark-gluon plasma (QGP) and induces a nonzero rotational motion [6-8]. The related global polarization of $\Lambda$ hyperons in relativistic heavy-ion collisions was measured by the STAR Collaboration [9]. They observed that the average vorticity of the QGP reaches $\omega \sim 10^{21} / \mathrm{s}$ making it the most vortical fluid in nature. Both electromagnetic and vorticity fields decay fast with the expansion of the hot medium $[4,5,8]$. One needs to find a sensitive probe to characterize the electromagnetic and vortical fields. Heavy quarks and especially quarkonia are the most sensitive and effective probes due to the following reasons: The heavy quark mass is much large than the QCD cutoff, $m_{c}, m_{b} \gg \Lambda_{Q C D}$, so their production can be well described by perturbative QCD; The heavy quark mass is much larger than the typical temperature of the hot medium, their mass does not change in the hot medium, and their number is conserved during the evolution; Heavy quarks are produced at a very early stage in the collision with a formation time of $\tau_{c} \sim 1 / 2 m_{c} \approx 0.06 \mathrm{fm} / \mathrm{c}$ for charm quark and $\tau_{b} \approx 0.02 \mathrm{fm} / \mathrm{c}$ for bottom quark, hence they can feel the strongest electromagnetic and vortical fields.

So far, many interesting topics related to heavy flavor in the electromagnetic and vortical fields emerge, see review paper [10]. One example is the photoproduction of heavy flavor mesons and quarkonia in peripheral and ultra-peripheral heavy-ion collisions $[11,12]$ that has been observed experimentally [13]. Heavy quarks in electromagnetic and vortical fields can generate directed flow $\left(v_{1}\right)[14,15]$. The slope of the $v_{1}$ rapidity dependence $\left(d v_{1} / d y\right)$

\footnotetext{
*e-mail: zhao-jx15@ tsinghua.org.cn
} 
of $D^{0}$ and $\bar{D}^{0}$ mesons is much larger than that for light hadrons $[16,17]$. The charmonium states will acquire non-collective elliptic flow $\left(v_{2}\right)$ in the strong magnetic field [18]. The directed flow of charmonium states is sensitive to the tilted initial energy profile that controls the magnitude of the global vorticity [19]. The static properties, e.g. mass and shape, of open/hidden heavy flavor states will be changed in electromagnetic fields [20-23]. Following these studies, we investigate charmonia in both electromagnetic and vortical fields [24].

For heavy flavor, there exists a hierarchy of scales: $m_{Q} \gg m_{Q} v \gg m_{Q} v^{2}$. Integrating out the degrees of freedom that are larger than $m_{Q}$ and $m_{Q} v$ sequentially in the QCD Lagrangian, one obtains the non-relativistic versions, NRQCD and pNRQCD [25, 26]. A potential model is derived by neglecting the interaction between color-singlet and color-octet states in the pNRQCD framework [26]. In this case, one can employ the Schrödinger equation to study the properties of heavy quarkonia. The Schrödinger equation has been successfully applied to heavy flavor states in vacuum and at finite temperature, see the review [10]. Heavy quarks in the rotating medium in electromagnetic fields can be described by the Dirac equation in the rotating frame,

$$
\left[i \gamma^{\mu}\left(\partial_{\mu}+i q A_{\mu}+\Gamma_{\mu}\right)-m\right] \Psi=0
$$

where $\Gamma_{\mu}$ is the affine connection, $A_{\mu}(x)$ the electromagnetic field in the rotating frame, and $q$ the charge of the quark [27]. It is straightforward to calculate the electromagnetic fields in the laboratory frame in heavy-ion collisions. One needs to transform the electromagnetic field between the rotating frame and laboratory frame. In our study, we consider a constant magnetic field along the rotating axis. Taking the symmetric gauge, the electromagnetic potential can be expressed as $A_{a}^{\prime}=\left(A_{0}^{\prime},-B y^{\prime} / 2, B x^{\prime} / 2,0\right)$ with the spacetime coordinates $\left(t^{\prime}, x^{\prime}, y^{\prime}, z^{\prime}\right)$ in the laboratory frame. The electromagnetic potential in the rotating frame can be obtained via $A_{\mu}=\left(A_{0}-\left(x^{2}+y^{2}\right) B \omega / 2,-B y / 2, B x / 2,0\right)$ with the coordinates $(t, x, y, z)$ in the rotating frame. In the non-relativistic limit and to first order of $1 / m$, we obtain the familiar one-body Schrödinger equation,

$$
\left[\frac{(\mathbf{p}-q \boldsymbol{A})^{2}}{2 m}-\frac{q}{m} \mathbf{B} \cdot \mathbf{s}-q A_{0}-\omega \cdot(\mathbf{s}+\mathbf{x} \times \mathbf{p})\right] \psi=E \psi .
$$

We can clearly see that there are no coupling terms between electromagnetic and vortical fields, which means that the rotation does not induce any electromagnetic effect. It is necessary to emphasize that the coupling term emerges if the electromagnetic fields are defined directly in the rotating frame [24].

We construct a two-body Schrödinger equation to describe a charmonium system in a rotational medium and under the impact of electromagnetic fields,

$$
\left[\frac{\left(\mathbf{p}_{a}-q_{a} \boldsymbol{A}_{a}\right)^{2}}{2 m_{a}}+\frac{\left(\mathbf{p}_{b}-q_{b} \boldsymbol{A}_{b}\right)^{2}}{2 m_{b}}-q_{a} \mathbf{E} \cdot \mathbf{x}_{a}-q_{b} \mathbf{E} \cdot \mathbf{x}_{b}-\omega \cdot\left(\mathbf{j}_{a}+\mathbf{j}_{b}\right)-\boldsymbol{\mu} \cdot \mathbf{B}+V\right] \psi=E \psi,
$$

where $\mathbf{j}_{i}=\mathbf{s}_{i}+\mathbf{x}_{i} \times \mathbf{p}_{i}$ is the total angular momentum that includes spin and orbital part, $-\boldsymbol{\mu} \cdot \mathbf{B}$ is the spin magnetic moment-magnetic field coupling term, and $m_{a}=m_{b}=m$ and $q_{a}=-q_{b}=q$ are the heavy quark mass and charge. The interaction potential $V$ between quark and antiquark contains a spin-spin interaction term and a spin-independent part. The latter becomes the Cornell potential in vacuum. When removing the electromagnetic(vorticity) related terms, we obtain the wave equation to study quarkonium in pure vortical(electromagnetic) field. Before solving Eq. 3, we first transform the single particle coordinates and momenta to the center-of-mass and relative ones, $\mathbf{R}=\left(\mathbf{x}_{a}+\mathbf{x}_{b}\right) / 2, \mathbf{r}=\mathbf{x}_{a}-\mathbf{x}_{b}, \mathbf{P}=\mathbf{p}_{a}+\mathbf{p}_{b}$, and $\mathbf{p}=\left(\mathbf{p}_{a}-\mathbf{p}_{b}\right) / 2$. Different from the case with only electromagnetic fields where the total wave function can always be separated into a center-of-mass and a relative part with the help of the conserved 
pseudo-momentum [21, 24], it is no longer possible to find a conserved quantity to separated the two parts in a rotational field. However, considering the fact that the strength of the magnetic field in relativistic heavy-ion collisions can reach $e B \approx 70 m_{\pi}^{2}$, which is much larger than the vortical field $m_{Q} \omega \approx m_{\pi}^{2}$ for charm quarks, we can choose the electromagnetic related parts as the main part and take the rotational related terms as perturbations. Therefore, the Hamiltonian can be expressed as,

$$
\begin{aligned}
H & =H_{\mathrm{EM}}+H^{\prime} \\
H_{\mathrm{EM}} & \equiv \frac{\mathbf{P}_{p s}^{2}}{4 m}+\frac{\mathbf{p}^{2}}{m}-\boldsymbol{\mu} \cdot \mathbf{B}-q \mathbf{E} \cdot \mathbf{r}-\frac{q}{2 m}\left(\mathbf{P}_{p s} \times \mathbf{B}\right) \cdot \mathbf{r}+\frac{q^{2}}{4 m}(\mathbf{B} \times \mathbf{r})^{2}+V, \\
H^{\prime} & \equiv-\omega \cdot\left(\mathbf{R} \times \mathbf{P}_{p s}\right)-\omega \cdot(\mathbf{l}+\mathbf{s})+\frac{q}{2} \omega \cdot(\mathbf{R} \times(\mathbf{B} \times \mathbf{r})),
\end{aligned}
$$

where $\mathbf{P}_{p s}=\mathbf{P}+q \mathbf{B} \times \mathbf{r} / 2$ is the pseudo-momentum which is the same as in pure electromagnetic fields. The contribution from the perturbation $H^{\prime}$ can be calculated through the standard method in quantum mechanics. The perturbation $H^{\prime}$ is relevant to the center-of-mass coordinate $\mathbf{R}$ hence the perturbative correction depends on the distance from the quarkonium to the rotating axis. That's the nature of particles in a vortical field and we can use the average value $\langle\mathbf{R}\rangle$ in our calculation. Details about the numerical calculation and results are shown in [24]. Here, we just show the effective potential in the two-body Schrödinger equation that can give us an intuitive understanding. Without the magnetic field, the potential (Cornell potential in
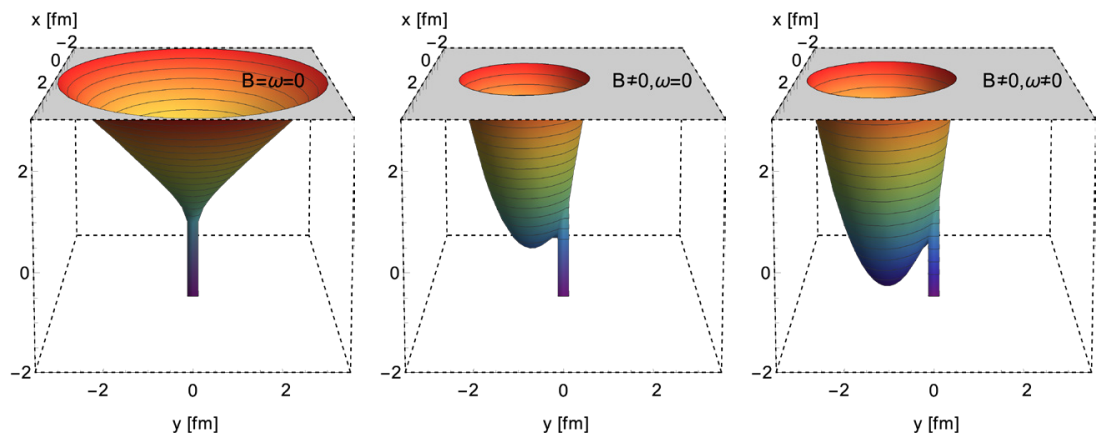

Figure 1. Spin-independent vacuum interaction potential between charm and anti-charm quark in $x-y$ plane with $z=0$ with/without magnetic and/or vortical fields. The unit of potential is $\mathrm{GeV}$.

vacuum) is a symmetric function in the $x-y$ plane, but the symmetry is broken when the magnetic field is turned on. When the magnetic field is strong enough, a new potential well forms and the $c \bar{c}$ bound state may tunnel from the strong interaction well to the magnetic well, as shown in Fig. 1. This is also known as Lorentz ionization [20]. The vortical field will deepen (shallow) the magnetic well. The vorticity will catalyze the transition from a strongly to an electromagnetically and rotationally interacting bound state.

In summary, we derived the non-relativistic wave equation under strong electromagnetic fields in the rotating frame. We constructed the two-body Schrödinger equation to investigated the static properties of charmonium states in strong electromagnetic and vortical fields. Since one usually defines the electromagnetic fields in the laboratory frame in heavy-ion collisions, there are no coupling terms between the magnetic and vortical fields in the Dirac and Schrödinger equations. The effective potential between charm and anti-charm quarks becomes anisotropic in electromagnetic and vortical fields, especially along the direction of the 
Lorentz force. The vorticity will increase (decrease) this asymmetry property. With increasing fields, the $c \bar{c}$ bound state which is confined by strong interaction in vacuum is gradually tunneled to an electromagnetic and vortical-field controlled anisotropic state. It is possible to realize this state in high-energy nuclear collisions. These anisotropic charmonium states will have a very different dynamic dissociation in the quark-gluon medium.

Acknowledgement: The work is supported by Guangdong Major Project of Basic and Applied Basic Research No. 2020B0301030008 and the NSFC under grant Nos. 11890712, 12075129, and 12047535 .

\section{References}

[1] D. E. Kharzeev, L. D. McLerran and H. J. Warringa, Nucl. Phys. A 803, 227-253 (2008).

[2] V. Skokov, A. Y. Illarionov and V. Toneev, Int. J. Mod. Phys. A 24, 5925-5932 (2009).

[3] V. Voronyuk, V. D. Toneev, W. Cassing, E. L. Bratkovskaya, V. P. Konchakovski and S. A. Voloshin, Phys. Rev. C 83, 054911 (2011).

[4] W. T. Deng and X. G. Huang, Phys. Rev. C 85, 044907 (2012).

[5] K. Tuchin, Adv. High Energy Phys. 2013, 490495 (2013).

[6] Z. T. Liang and X. N. Wang, Phys. Rev. Lett. 94, 102301 (2005) [erratum: Phys. Rev. Lett. 96, 039901 (2006)].

[7] F. Becattini, F. Piccinini and J. Rizzo, Phys. Rev. C 77, 024906 (2008).

[8] Y. Jiang, Z. W. Lin and J. Liao, Phys. Rev. C 94, no.4, 044910 (2016) [erratum: Phys. Rev. C 95, no.4, 049904 (2017)].

[9] L. Adamczyk et al. [STAR], Nature 548, 62-65 (2017).

[10] J. Zhao, K. Zhou, S. Chen and P. Zhuang, Prog. Part. Nucl. Phys. 114, 103801 (2020).

[11] W. Zha, L. Ruan, Z. Tang, Z. Xu and S. Yang, Phys. Lett. B 789, 238-242 (2019).

[12] W. Shi, W. Zha and B. Chen, Phys. Lett. B 777, 399-405 (2018).

[13] J. Adam et al. [ALICE], Phys. Rev. Lett. 116, no.22, 222301 (2016).

[14] S. Chatterjee and P. Bożek, Phys. Rev. Lett. 120, no.19, 192301 (2018).

[15] S. K. Das, S. Plumari, S. Chatterjee, J. Alam, F. Scardina and V. Greco, Phys. Lett. B 768, 260-264 (2017).

[16] J. Adam et al. [STAR], Phys. Rev. Lett. 123, no.16, 162301 (2019).

[17] S. Acharya et al. [ALICE], Phys. Rev. Lett. 125, no.2, 022301 (2020).

[18] X. Guo, S. Shi, N. Xu, Z. Xu and P. Zhuang, Phys. Lett. B 751, 215-219 (2015).

[19] B. Chen, M. Hu, H. Zhang and J. Zhao, Phys. Lett. B 802, 135271 (2020).

[20] K. Marasinghe and K. Tuchin, Phys. Rev. C 84, 044908 (2011).

[21] J. Alford and M. Strickland, Phys. Rev. D 88, 105017 (2013).

[22] S. Cho, K. Hattori, S. H. Lee, K. Morita and S. Ozaki, Phys. Rev. Lett. 113, no.17, 172301 (2014).

[23] S. Iwasaki, M. Oka and K. Suzuki, Eur. Phys. J. A 57, no.7, 222 (2021).

[24] S. Chen, J. Zhao and P. Zhuang, Phys. Rev. C 103, no.3, L031902 (2021).

[25] W. E. Caswell and G. P. Lepage, Phys. Lett. B 167, 437-442 (1986).

[26] N. Brambilla, A. Pineda, J. Soto and A. Vairo, Nucl. Phys. B 566, 275 (2000).

[27] H. L. Chen, K. Fukushima, X. G. Huang and K. Mameda, Phys. Rev. D 93, no.10, 104052 (2016). 